\title{
New configuration for efficient and durable copper coating on the outer surface of a tube
}

\author{
Irfan Ahmad, Steven F. Chapman, Katherine M. Velas, and Mahadevan Krishnan \\ Alameda Applied Sciences Corporation (AASC), San Leandro, California 94577, USA
}

(Received 5 October 2016; published 27 March 2017)

\begin{abstract}
A well-adhered copper coating on stainless steel power coupler parts is required in superconducting radio frequency (SRF) accelerators. Radio frequency power coupler parts are complex, tubelike stainless steel structures, which require copper coating on their outer and inner surfaces. Conventional copper electroplating sometimes produces films with inadequate adhesion strength for SRF applications. Electroplating also requires a thin nickel strike layer under the copper coating, whose magnetic properties can be detrimental to SRF applications. Coaxial energetic deposition (CED) and sputtering methods have demonstrated efficient conformal coating on the inner surfaces of tubes but coating the outer surface of a tube is challenging because these coating methods are line of sight. When the substrate is off axis and the plasma source is on axis, only a small section of the substrate's outer surface is exposed to the source cathode. The conventional approach is to rotate the tube to achieve uniformity across the outer surface. This method results in poor film thickness uniformity and wastes most of the source plasma. Alameda Applied Sciences Corporation (AASC) has developed a novel configuration called hollow external cathode CED (HEC-CED) to overcome these issues. HEC-CED produces a film with uniform thickness and efficiently uses all eroded source material. The $\mathrm{Cu}$ film deposited on the outside of a stainless steel tube using the new HEC-CED configuration survived a high pressure water rinse adhesion test. HEC-CED can be used to coat the outside of any cylindrical structure.
\end{abstract}

DOI: 10.1103/PhysRevAccelBeams.20.033502

\section{INTRODUCTION}

Delivering RF power from room temperature to the inside of cryogenic superconducting radio frequency (SRF) accelerator cavities requires a power coupler to be thermally isolating but electrically conducting. Stainless steel (ss) parts coated with a few skin depths of copper can meet these conflicting requirements [1-3]. Electroplating is a popular low cost method for copper coating but inadequate adhesion of the electroplated copper on the stainless steel coupler parts can be a problem. Electroplating also requires a nickel strike layer [4], which is not desirable because it is magnetic and can be passivated, precluding $\mathrm{Cu}$ plating [5].

Coaxial energetic deposition (CED) is a cathodic arc coating method in which metal plasma with high ion energies $(60-120 \mathrm{eV})$ is generated from a metal cathode [6]. Arc spots with lifetimes on the order of hundreds of nanoseconds form and annihilate throughout the duration of a single pulse, which typically lasts hundreds of milliseconds. The path traversed by the arc spots on the cathode is

\footnotetext{
*orresponding author. irfanplsr@gmail.com

Published by the American Physical Society under the terms of the Creative Commons Attribution 4.0 International license. Further distribution of this work must maintain attribution to the author(s) and the published article's title, journal citation, and DOI.
}

microscopically fractal but globally helical in the presence of an external magnetic field [7,8]. Collectively, these spots form an expanding spoke of plasma ejected normal to the surface of the cathode. The highly energetic ions hit the substrate and penetrate a few monolayers below the surface, resulting in a film with high adhesion strength. A uniform film is deposited on a substrate using thousands of pulses. The acceptable range of residual resistivity ratio (RRR) values for copper coating on coupler parts by European X-ray Free Electron Laser (E-XFEL) standards is 30-80 [9]. CED coated copper films have demonstrated a RRR of 50 and high adhesion strength on ss substrates [10]. In addition, CED Nb films have demonstrated early success in SRF applications, including high crystallinity [11,12], a record RRR [11], and strong adhesion strength [12].

The warm inner conductor (WIC) of the rf coupler assembly requires a coating on its outer surface. CED has demonstrated high adhesion strength copper coatings on the inner surface of ss tubes. However, coating the outside requires complicated hardware to rotate the substrate and wastes most of the eroded cathode material. This paper presents an efficient configuration for coating the outer surface of a cylindrical substrate using cathodic arc deposition. First, we describe the hollow external cathode CED (HEC-CED) configuration. Unlike conventional CED, this configuration does not require any moving or rotating parts to produce a uniform coating on the outside of a tube. In addition, the eroded cathode material is fully utilized. 
Second, we discuss the arc physics in the HEC-CED configuration. Third, we describe the potential of HECCED to coat rf coupler parts or any arbitrarily shaped object. Lastly, we present a comparison of HEC-CED with the original CED configuration and highlight the benefits of HEC-CED.

The new HEC-CED configuration demonstrated successful coating of a mock warm inner conductor (WIC) power coupler part. The coated mock coupler part was subjected to a $1500 \mathrm{psi}\left(1.03 \times 10^{7} \mathrm{~Pa}\right)$ high-pressure water rinse (HPR) for ten minutes. The copper coating remained adhered to the tube.

\section{THE HEC-CED CONFIGURATION}

An illustration of the HEC-CED configuration is shown in Fig. 1. A large hollow cathode is coaxial with a smaller outer diameter (OD) substrate, which acts as an anode. The arc is initiated on the inner surface of the hollow cathode at the trigger plane (see Fig. 2). The average arc current is approximately $150 \mathrm{~A}$, but this current is carried by multiple cathode spots, each carrying a fraction of the current on $100 \mathrm{~ns}$ time scales before extinguishing [13]. This series of arc spots produces a dendritic path on the cathode that, in the presence of an external magnetic field, helically wraps around the cathode surface. Random variation in the arc path on each shot leads to plasma uniformly coating the outer surface of the anode with energetic copper ions, when integrated over thousands of shots. As the stochastic paths of the arc spots address all azimuthal and longitudinal locations equally, this new configuration has no need for mechanically rotating parts.

All tests described in this paper were performed in the CED-3 coating chamber, which consists of a $160 \mathrm{~cm}$ long, double walled, vertical spool with $25 \mathrm{~cm}$ diameter con-flat (CF) flanges at either end. The spool is mounted on a CF tee, which has ports for pumps and gauges. The base

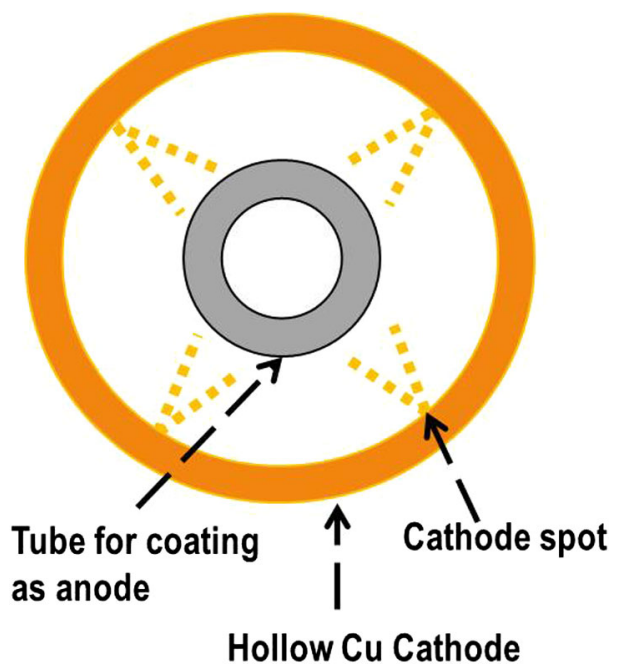

FIG. 1. Concept of the HEC-CED coating configuration.

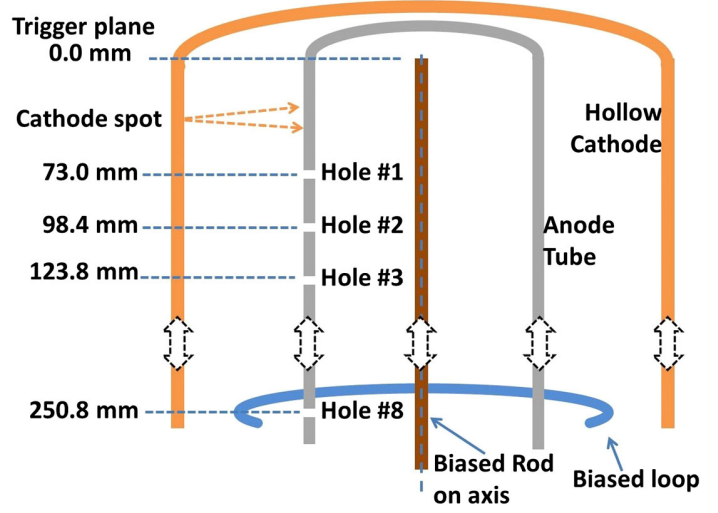

FIG. 2. Schematic of a section of the $\mathrm{v}_{z}$ measurement arrangement in the HEC-CED configuration.

pressure of the system is in the low $10^{-8}$ Torr $\left(1.3 \times 10^{-8}\right.$ mbar $)$ range. A custom made $\mathrm{CF}$ flange at the top of the spool has cathode, anode, trigger, thermocouple and heater feedthroughs. The spool has a solenoid wound on the outer wall to create the axial magnetic field that results in the helical path of arc spots on the cathode surface. The solenoid is driven by a current of $30 \mathrm{~A}$ to create a $5.6 \mathrm{mT}$ field on axis. A pulse-forming network (PFN) is used to trigger the arc. The PFN discharge provides a $200 \mu$ s pulse with a peak current of $260 \mathrm{~A}$, which creates the initial seed plasma necessary for arc breakdown. Arc current is supplied by a $200 \mathrm{~A}, 100 \mathrm{~V}$ power supply. The arc running voltage is typically $30 \mathrm{~V}$ and the current is in the 145-165 A range.

\section{A. Spoke velocity measurement in HEC-CED}

In order to characterize the arc spot behavior in the new configuration, an anode tube with eight holes of $3.3 \mathrm{~mm}$ diameter, $25.4 \mathrm{~mm}$ apart was built to measure the apparent helical velocity of the plasma spoke. This apparent velocity corresponds to a phase velocity of the arc spots as they extinguish and are reborn elsewhere along the surface of the cathode. A schematic of the arrangement is shown in Fig. 2. The anode fits inside a $5 \mathrm{~cm}$ inner diameter (ID) $\mathrm{Cu}$ cathode. The first and eighth holes were 73 and $250.8 \mathrm{~mm}$ below the trigger plane, respectively. A $6.4 \mathrm{~mm}$ diameter copper rod was placed on the axis. A $41 \mathrm{~mm}$ diameter loop of $1.15 \mathrm{~mm}$ diameter copper wire was placed in the plane of the last hole between the anode and the cathode, as shown in the figure. Both the central rod and the wire loop were biased to $-20 \mathrm{~V}$ to measure the ion current from the arc spoke plasma. These were biased from a common capacitor bank ( $40.8 \mathrm{mF}, 350 \mathrm{~V})$ that supplied the charge necessary to maintain the bias voltage throughout the duration of the arc pulse.

Data from a typical arc pulse are presented in Fig. 3. The signal on the biased central rod [Fig. 3(a)] is a regular series of sharp spikes. The signal on the biased loop [Fig. 3(b)] is a set of broad peaks near the end of the event. The data from 


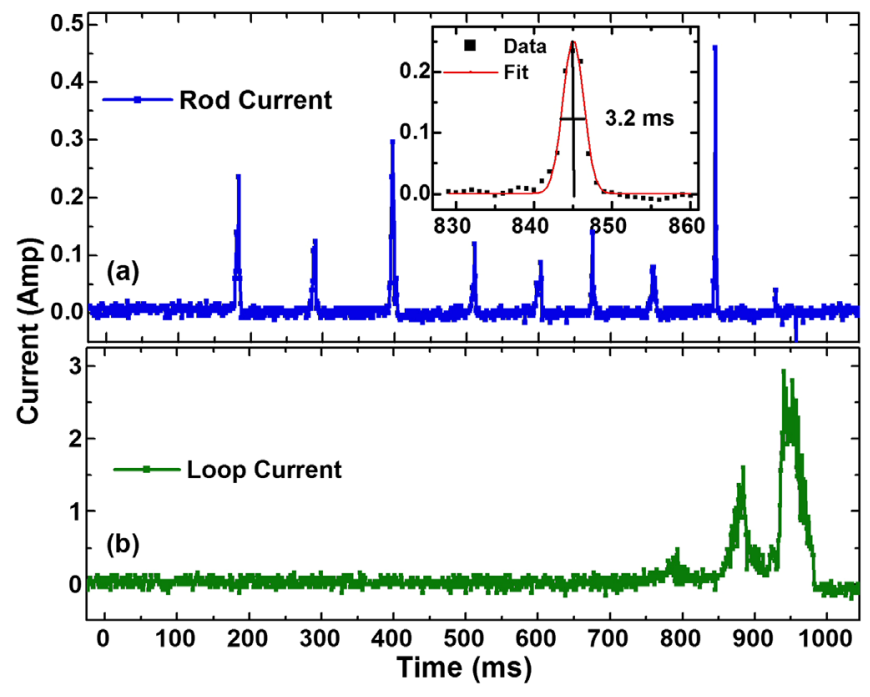

FIG. 3. Typical data from an arc pulse: (a) the data from the biased rod, and (b) from the biased loop. The inset in (a) is a twopoint average of the rod current data around the peak at $845 \mathrm{~ms}$ with peak fitting.

the biased rod shows peaks at $183,290,397,511,603,675$, 759 , and $845 \mathrm{~ms}$, corresponding to holes \#1 through hole $\# 8$, respectively. Because the holes are $2.54 \mathrm{~cm}$ apart, the average axial spoke velocity, $\mathrm{v}_{z}$, is $(28.8 \pm 6.5) \mathrm{cm} / \mathrm{s}$. The first peak in the loop data at $883 \mathrm{~ms}$ gives the time the spoke took to cover a vertical distance of $25.1 \mathrm{~cm}$, indicating an apparent spoke $\mathrm{v}_{z}$ of $28.4 \mathrm{~cm} / \mathrm{s}$. The second large broad peak from the loop at $950 \mathrm{~ms}$ is from the spoke dwelling at the bottom of the cathode before the arc power supply is turned off. By comparison, the measured spoke $\mathrm{v}_{z}$ in the original CED configuration is approximately $67 \mathrm{~cm} / \mathrm{s}$, on a $0.95 \mathrm{~cm}$ diameter copper cathode.

The (apparent) angular speed of the arc, $\omega$ is determined as follows. A $3.3 \mathrm{~mm}$ hole on a $16 \mathrm{~mm}$ radius tube subtends a $0.206 \mathrm{rad}\left(11.8^{\circ}\right)$ angle to the axis. The data in Fig. 3 show the largest rod current peak is centered at $845 \mathrm{~ms}$. This peak was smoothed with adjacent, two-point averaging and fit to a Gaussian curve, as shown in the inset of Fig. 3. The full width at half maximum of the peak is $3.2 \mathrm{~ms}$. The vertical distance traveled during this period with an average $\mathrm{v}_{z}$ of $28.4 \mathrm{~cm} / \mathrm{s}$ is $0.91 \mathrm{~mm}$, which is smaller than the hole diameter of $3.3 \mathrm{~mm}$. Thus, we conclude that the width of the peak is due to the angular transit time of the arc spot. With $3.2 \mathrm{~ms}$ to cross the 3.3 diameter hole on a tube with $32 \mathrm{~mm} \mathrm{OD}$, the estimated angular frequency is approximately $64.5 \mathrm{rad} / \mathrm{s}$. In the CED configuration, the angular frequency measured $52 \mathrm{~mm}$ away from a $0.95 \mathrm{~cm}$ OD Cu cathode was approximately $345 \mathrm{rad} / \mathrm{s}$, at the same applied magnetic field.

Table I summarizes key parameters in the CED and HEC-CED configurations. The HEC-CED angular frequency is about $20 \%$ of the CED angular frequency. However, the azimuthal speed, $\mathrm{v}_{\theta}$, is the same in both
TABLE I. Comparison of parameters in the CED versus HEC-CED configuration.

\begin{tabular}{lccc}
\hline \hline Parameter & CED & HEC-CED & HEC-CED /CED \\
\hline Cathode diameter $(\mathrm{cm})$ & 0.95 & 5.08 & 5.3 \\
Angular frequency $\omega(\mathrm{rad} / \mathrm{s})$ & 345 & 64.5 & $1 / 5.4$ \\
Azimuthal speed $\mathrm{v}_{\theta}(\mathrm{cm} / \mathrm{s})$ & 164 & 164 & 1 \\
Axial speed $\mathrm{v}_{z}(\mathrm{~cm} / \mathrm{s})$ & 67 & 28.4 & 0.43 \\
\hline \hline
\end{tabular}

configurations. Consider the physics of arc spots forming in a given spot, then extinguishing and being reborn at an adjacent location. Published values for the typical arc spot lifetime in $\mathrm{Cu}$ are $95 \mathrm{~ns}$ [13]. The common azimuthal speed (for both cathodes, see Table I), multiplied by this lifetime gives a spot-to-spot hopping distance of $0.16 \mu \mathrm{m}$. This suggests a smaller spot size, such as $0.1 \mu \mathrm{m}$. A sheath voltage of $20 \mathrm{~V}$ across this diameter gives an electric field of $2 \times 10^{8} \mathrm{~V} / \mathrm{m}$. Such fields are capable of supporting field enhanced thermionic emission from an arc spot with sufficient current density to carry $\sim 10-50$ A. For a fixed axial magnetic field, the arc spot physics should be the same on cathodes of different size; hence the spot to spot distance and, therefore, $\mathrm{v}_{\theta}$ should be the same, as observed. However, the angular frequency would vary inversely with cathode radius, as also observed in Table I.

The axial speed, $\mathrm{v}_{z}$ is reduced to $28.4 \mathrm{~cm} / \mathrm{s}$ in the HECCED configuration from $67 \mathrm{~cm} / \mathrm{s}$ in the CED configuration with a 5 times larger diameter cathode. This difference may simply be because the larger cathode provides a larger surface area for arc spots to form upon. The arc takes more time to traverse the larger angular space and the apparent axial velocity is reduced. Further experiments are required to investigate this hypothesis.

\section{B. Coating of mock WIC_2 with HEC-CED}

To demonstrate the potential of HEC-CED to coat coupler parts, a mock WIC (WIC_2) modeled after an actual Tesla Test Facility (TTF) WIC was designed. The mock part nearly matches the OD, ID, and end flange dimensions of the TTF WIC. The WIC_2 substrate preparation was a sequential, three-step chemical cleaning using trichloroethylene, acetone, and isopropyl alcohol, followed by drying the substrate with $\mathrm{N}_{2}$. After cleaning, the substrate was loaded into the coating chamber, which was baked at $120-150^{\circ} \mathrm{C}$ for two days. A 2000 Watt, 10" long cartridge heater was placed inside the WIC_2 tube to heat the substrate to $270{ }^{\circ} \mathrm{C}$ during the entire baking and coating process. A $30 \mu \mathrm{m}$ copper film was deposited, to meet E-XFEL standards.

Figure 4(a), shows the coated mock WIC_2 next to an actual TTF WIC for comparison. The hollow $26 \mathrm{~cm}$ long, $5 \mathrm{~cm}$ ID copper cathode is also shown. On WIC_2, there is a greyish ring $26 \mathrm{~cm}$ from the top, which is due to partial erosion from the ss holder of the copper cathode. This issue is easily avoidable by designing an extra margin in the 


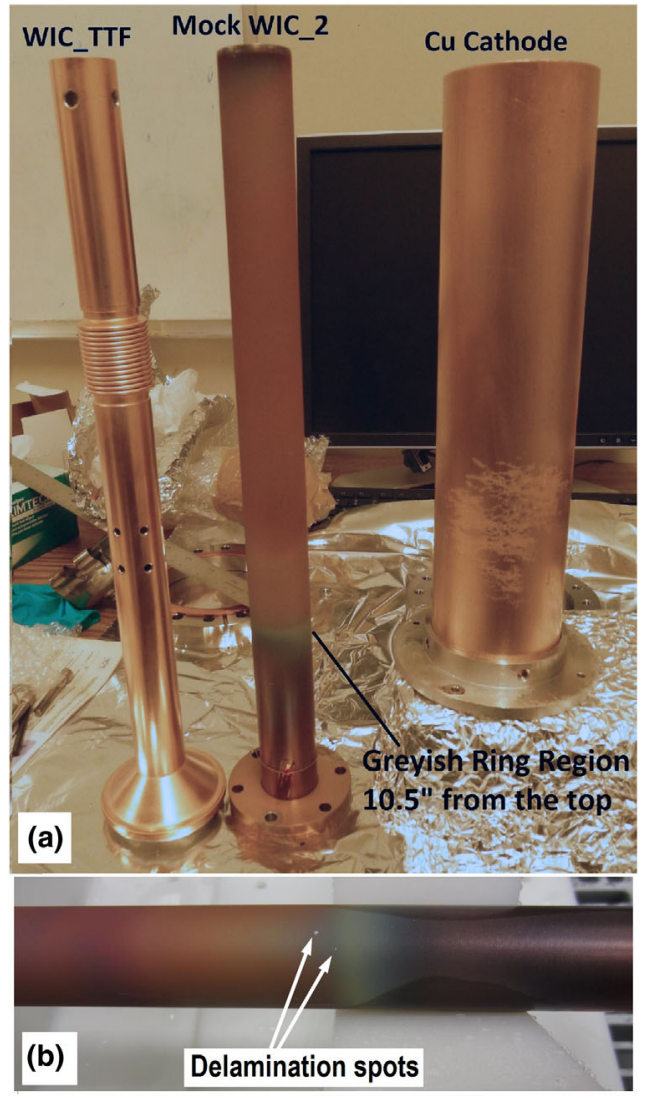

FIG. 4. Coated mock WIC_2: (a) next to a TTF WIC, before the HPR test, and (b) after the test.

cathode length. There is also a discoloration in the middle of the tube because the chamber was vented while this region was hotter than $150^{\circ} \mathrm{C}$.

After coating, the mock WIC_2 was sent to Fermi National Accelerator Laboratory (FNAL) for adhesion testing. Several methods are employed to gauge the adhesion strength of the copper, including the HPR test and the cold shock test. In the HPR test, high pressure, particulate free water exiting from a narrow nozzle (2-3 $\mathrm{mm}$ diameter) is sprayed directly on to the coated film. The distance between the substrate and nozzle can be varied and the pressure of the water at the coated film can be estimated. In the cold shock test, the coated parts are submerged in a liquid nitrogen bath for a few hours, taken out, left to reach room temperature, and inspected for delamination. If the coated film is not well adhered to the substrate, it partially (or fully) delaminates due to different thermal expansion coefficients of the film material and the substrate.

Figure 4(b), shows the mock WIC_2 after the HPR test. The mock WIC_2 was subjected to the HPR test using $1500 \mathrm{psi}\left(1.03 \times 10^{7} \mathrm{~Pa}\right)$ water. There was no delamination of the $\mathrm{Cu}$ coating. There were a few delamination spots in the greyish region which had stainless steel contamination from the anode holder. This demonstrated the use of
HEC-CED to coat the outer surface of cylindrical hardware with a well-adhered $\mathrm{Cu}$ film.

\section{Coating shapes other than a right cylinder}

This section presents a strategy for uniform coating of shapes more complex than right circular cylinders. There are two issues to consider: (1) the ion flux decreases away from the cathode surface, so the cathode radius must be adjusted to compensate for varying substrate radius; (2) the axial propagation speed of the cathode spots also varies with cathode radius, and so does the flux of ions to the substrate. These effects are simple to compensate for, as long as the substrate is large enough to intercept all the ions from any given cathode spot. Figure 5 shows the concept.

The arc spokes (two are shown here) travel over the cathode inner surface, with a varying axial velocity, $\mathrm{v}_{z}$, and a fixed angular velocity, $\mathrm{v}_{\theta}$. The plasma from a given spot expands into a cone of roughly $15^{\circ}$ half angle (a Mach 4 expansion) and the flux decreases as the distance from the cathode surface squared. We can calculate a radial distance between the cathode and the substrate at any location such that the incident flux gives a constant film thickness. Such a calculation must also take into account the variation in axial speed. Constant film thickness demands that $\left(\mathrm{r}_{2} / \mathrm{r}_{1}\right)=\left(\mathrm{v}_{z 1} / \mathrm{v}_{z 2}\right)$. Only two measurements were made of this variation, for two cathode radii. The speed decreased from $67 \mathrm{~cm} / \mathrm{s}$ for $\mathrm{r}_{1}=0.95 \mathrm{~cm}$ to $28.4 \mathrm{~cm} / \mathrm{s}$ for $\mathrm{r}_{2}=$ $5.08 \mathrm{~cm}$ (Table I). To calculate the desired cathode shape for an arbitrary substrate, one must measure $\mathrm{v}_{z}$ for several radii and fit the data (such as to a polynomial fit). The cathode radius at any axial location may then be estimated as above.

For the specific case of the TTF WIC substrate, it was not possible to calculate the cathode shape precisely, because only two values of $\mathrm{v}_{z}$ were available. The cathode surface area increases with radius, hence the available area at any axial location to be covered by spots increases, thereby reducing the axial speed, as observed. A straight line extrapolation of the two data points would lead to a

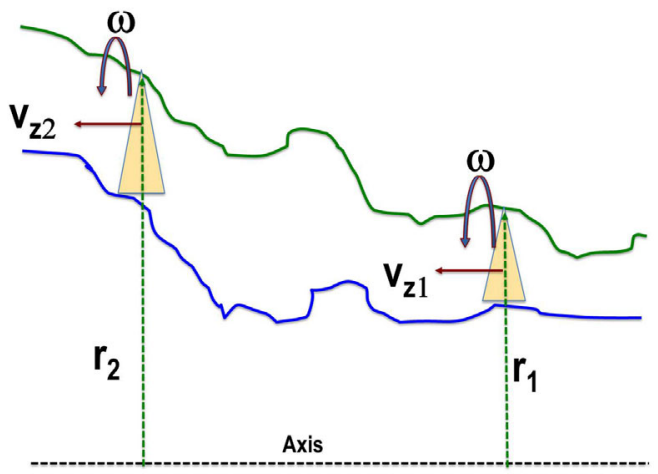

FIG. 5. Illustration of the concept for using a shaped cathode in HEC-CED to achieve a uniform film thickness on an arbitrarily shaped substrate. 


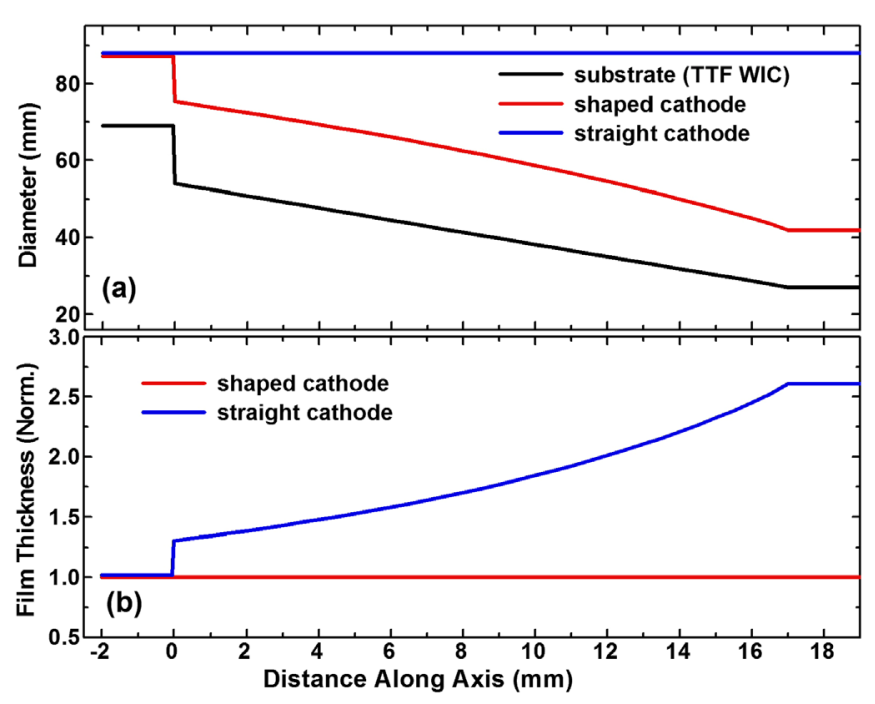

FIG. 6. (a) The diameter profiles of a straight cathode (blue), a shaped cathode (red) and the TTF WIC part (black). (b) Thickness variation (normalized) for a straight cathode (blue) vs a variable cathode (red).

negative axial velocity beyond a certain radius, which is not physical. An exponential fit was selected, as it is consistent with the physical notion that the axial speed must decrease asymptotically to a finite (positive) value as the cathode radius is increased.

Figure 6(a) shows three profiles: the TTF WIC part to be coated (black), the shaped cathode (red) and the straight cathode (blue). Figure 6(b) shows the estimated film thickness, normalized to its value at the largest radius.

For the straight cathode (blue) the thickness increases by a factor of $>2.5$ as the diameter gets smaller (emitted mass covers a smaller area). With the compensated, shaped cathode the thickness remains the same everywhere. At the abrupt step in the diameter of the WIC part, some ions will be at grazing incidence and might not adhere. But, as the plasma arrives at the surface in a divergent cone, the effect should be small.

\section{COMPARISON OF HEC-CED AND CED CONFIGURATIONS}

In this section, we describe the conventional CED method, its effectiveness in coating the inside surface of a tube and ineffectiveness in coating the outer surface of a tube. Finally, we compare both configurations' capacity to coat the outer surface of a tube. Figure 7(a) shows the CED configuration. The arc is triggered between a centrally placed cathode rod and a coaxial cylindrical anode mesh. When coating the inner surface of a tube, the substrate may act as the anode and the random helical pattern of arc spots (integrated over thousands of shots) results in a uniform coating on the inner surface of the anode.

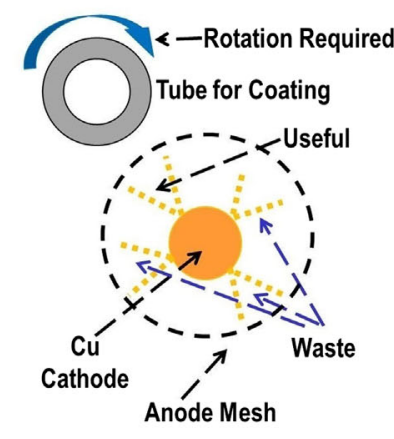

(a)
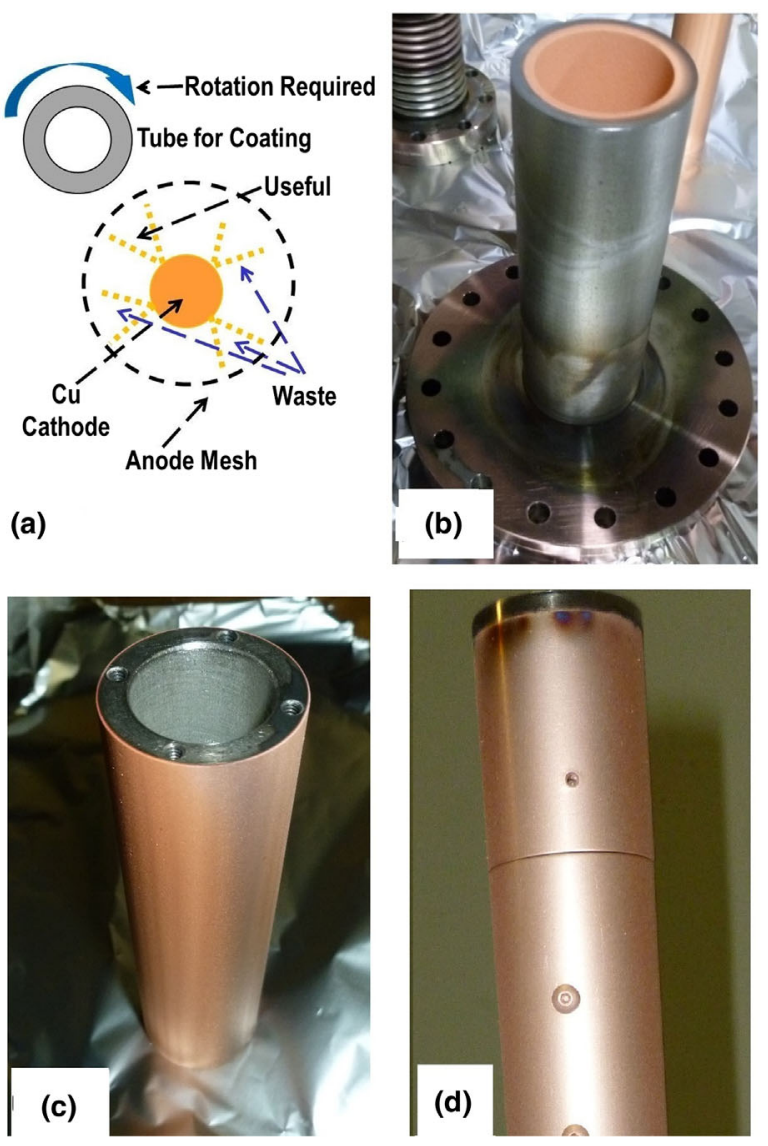

FIG. 7. (a) Arrangement for coating the outer surface of a tube with CED, (b) coating the inside surface of a tube with CED, (c) coating the outer surface of a tube with CED, and (d) coating of the outer surface of a tube with HEC-CED.

Figure 7(b) shows a mock cold external conductor (CEC), a part from the E-XFEL rf coupler that was coated with a $62 \mu \mathrm{m}$ copper film using conventional CED. The mock CEC nearly matches the length, ID, and end flange of the TTF CEC coupler part. The mock CEC, which served as the anode, was $360 \mathrm{~mm}$ long with ID of $38 \mathrm{~mm}$. A visual inspection revealed a uniform copper coating on the mock CEC. At FNAL, the coating on the mock CEC passed both the HPR $\left(1200 \mathrm{psi}, 8.27 \times 10^{6} \mathrm{~Pa}\right)$ and the cold shock (77 K) tests.

To coat the external surface of the WIC using CED, the substrate was placed outside of an anode mesh, as shown in Fig. 7(a). A small fraction of the plasma expanding beyond the anode lands on the outer surface of the tube.

Figure 7(c) shows a mock WIC (WIC_1) after coating with $30 \mu \mathrm{m}$ of copper, using CED. The mock WIC_1 was $150 \mathrm{~mm}$ long with an OD of $32 \mathrm{~mm}$, matching the OD of the TTF WIC. WIC_1 was connected to a manual rotation feedthrough. Only a small section of the substrate has a line of sight to the cathode to receive $\mathrm{Cu}$ ions, so the substrate must be rotated to expose the entire surface to the incident plasma. The degree of coating uniformity is dependent on the number of positions on the circumference of the 
TABLE II. The comparison of conventional vs new configuration.

\begin{tabular}{lcc}
\hline \hline Characteristiclconfiguration & HEC-CED & CED \\
\hline Rotation requirement & No & Yes \\
Additional mesh requirement & No & Yes \\
$\%$ waste material & $<10 \%$ & $90 \%$ \\
Coating duration & $\mathrm{t}$ & $10 \mathrm{t}$ \\
Coating adhesion & Strong & Poor \\
\hline \hline
\end{tabular}

tube used for coating. WIC_1 was coated using four positions, $90^{\circ}$ apart. At each location, a $15 \mu \mathrm{m}$ film was deposited before rotating the substrate. The process was repeated to achieve a $30 \mu \mathrm{m}$ coating on the WIC_1 tube in eight steps.

A visual inspection [Fig. 7(c)] revealed that the coating on WIC_1 was nonuniform with vertical stripes. The nonuniform copper film on WIC_1 is a result of the stepwise coating process. Using more deposition positions can reduce the nonuniformity on the outer surface coating. However, even with many rotations, only limited coating uniformity can be achieved because the divergent plasma plume deposits a film with varying thickness on the cylindrical surface. The mock WIC_1 tube passed the thermal shock test but failed the HPR test. During the HPR test, the $\mathrm{Cu}$ film peeled off in two separate layers. To contrast, a tube coated using HEC-CED is presented in Fig. 7(d). This tube shows superior coating uniformity.

There are two clear advantages to using HEC-CED instead of conventional CED to coat the outer surface of a cylindrical structure; there is no rotating hardware and almost no wasted cathode material. The CED configuration needs a mesh anode and half of the cathode material is deposited on the $50 \%$ transparent mesh. Of the plasma that passes through the anode, a small fraction $(20 \%)$ of the material reaches the outer surface of the substrate, as depicted in Fig. 7(a). Therefore, only approximately $10 \%$ of the eroded material is deposited on the substrate. In HEC-CED, almost all of the material eroded from the outer hollow cathode hits the inner anode substrate. The difference in plasma utilization means the CED configuration needs 10 times the number of pulses to get the same coating as in HEC-CED. Furthermore, the adhesion of films deposited using HEC-CED is better than that of films deposited in multiple steps using CED. Combining these benefits makes HEC-CED superior to the conventional CED configuration for coating the outer surface of a tube. All of these advantages are summarized in Table II.

\section{SUMMARY}

We have presented a more efficient configuration for coating the outer surface of a tube using the CED process. The HEC-CED configuration is demonstrated to reduce drastically the coating duration and coating material waste as compared to conventional CED. The new configuration does not require moving parts. The apparent phase velocity of the arc spots on the inside of a large diameter hollow cathode is measured and compared with that in the conventional configuration. Strong adhesion of the coated copper film on stainless steel tubes is demonstrated. Finally, the potential of the new configuration to uniformly coat complex cylindrical shapes is explored. HEC-CED is a novel process that efficiently coats the outside of cylindrical objects. Using both CED and HEC-CED, all components of stainless steel SRF power couplers can be efficiently coated with a durable $\mathrm{Cu}$ film. This process can be used to coat the outside of any cylindrical metal object with any metallic film.

\section{ACKNOWLEDGMENTS}

This research is supported at AASC by the U.S. Department of Energy via Grant No. DE-SC0009581. The authors gratefully acknowledge A. C. Crawford at FNAL for the HPR and cold shock tests as well as many fruitful discussions. We also acknowledge receiving TTF coupler parts from and productive discussions with Dr. W. Kaabi of LAL, Orsay, France.

[1] M. Fouaidy and N. Hammoudi, RRR of copper coating and low temperature electrical resistivity of material for TTF couplers, Physica (Amsterdam) 441C, 137 (2006).

[2] T. Treado and S. Einarson, in Proceedings of the 22nd Particle Accelerator Conference, PAC-2007, Albuquerque, NM (IEEE, New York, 2007), THIBKI02.

[3] X. Singer, H. M. Wen, W. Singer, and W. D. Moller, Properties and structure of electrodeposited copper layers in parts of the TTF main coupler, in Proceedings of the 11th Workshop on rf Superconductivity, Lubeck, Germany, 2011, THP18.

[4] L. Popielarski, M. Hodek, M. Goodrich, I. Malloch, N. Putman, R. Oweiss, J. Popielarski, K. Saito, and D. Victory, Testing of copper plating quality on REA3 coupler bellows and approach to improved plating for FRIB production, in Proceedings of SRF, Paris, France, 2013, THP067.

[5] A. Balasubramanian, D. S. Srikumar, G. Raja, G. Saravanan, and S. Mohan, Effect of pulse parameter on pulsed electrodeposition of copper on stainless steel, Surf. Eng. 25, 389 (2009).

[6] M. Krishnan, E. Valderrama, C. James, X. Zhao, J. Spradlin, A-M Valente Feliciano, L. Phillips, C. E. Reece, K. Seo, and Z. H. Sung, Energetic condensation growth of Nb thin films, Phys. Rev. ST Accel. Beams 15, 032001 (2012).

[7] R. P. Welty, Apparatus and method for coating a substrate using vacuum arc evaporation, U.S. Patent No. 5,269,898 (1993).

[8] A. Anders, Cathodic Arcs: From Fractal Spots to Energetic Condensation (Springer, New York, 2008), Vol. 50.

[9] S. Einarson and T. Treado, Power coupler manufacturing and quality control at CPI, in Proceedings of IPAC, Dresden, Germany, 2014, WEPME024. 
[10] I. Irfan, M. Krishnan, S. F. Chapman, K. M. Velas, and W. Kaabi, Energetic copper coating on stainless steel power couplers for SRF application, in Proceedings of SRF, Whistler, Canada, 2015, THPB083.

[11] M. Krishnan, E. Valderrama, B. Bures, K. Wilson-Elliott, X. Zhao, L. Phillips, A.-M. Valente-Feliciano, J. Spradlin, C. Reece, and K. Seo, Very high residual resistivity ratios of hetero-epitaxial superconducting niobium films on $\mathrm{MgO}$ substrates, Supercond. Sci. Technol. 24, 115002 (2011).
[12] M. Krishnan, I. Irfan, T. Tajima, T. Proslier, R. Geng, P. Kneisel, C. Reece, and X. Zhao, Energetic condensation growth of niobium films, in Proceedings of SRF, Paris, France, 2013, WEIOA02.

[13] A. Anders, S. Anders, B. Juttner, H. Pursch, H. Botticher, and H. Luck, Vacuum arc cathode spot parameters from high-resolution luminosity measurements, J. Appl. Phys. 71, 4763 (1992). 